BULL. AUSTRAL. MATH. SOC.

VOL. $3(1970), 111-123$.

\title{
Absolute Nörlund summability of Fourier series of functions of bounded variation
}

\section{Masako Izumi and Shin-ichi Izumi}

\begin{abstract}
The authors prove two theorems. The first theorem generalizes theorems due to T. Singh and O.P. Varshney, concerning absolute Nörlund summability of Fourier series of functions of bounded variation. The second theorem generalizes theorems of L.S. Bosanquet and H.P. Dikshit.
\end{abstract}

\section{Introduction and theorems}

1.1. Let $\sum a_{n}$ be an infinite series and $\left(s_{n}\right)$ be the sequence of its partial sums. Let $\left(p_{n}\right)$ be a sequence of positive numbers and let $P_{n}=p_{0}+p_{1}+\ldots+p_{n}$ for $n \geqq 0$ and $p_{-1}=P_{-1}=0$. We suppose that $P_{n}+\infty$ as $n \rightarrow \infty$. The sequence $\left(t_{n}\right)$ defined by

$$
t_{n}=\frac{1}{p_{n}} \sum_{k=0}^{n} p_{n-k} s_{k} . \quad(n=1,2, \ldots)
$$

is called the Nörlund means of the series $\sum a_{n}$. If the sequence $\left(t_{n}\right)$ is of bounded variation, that is, $\sum\left|t_{n}-t_{n-1}\right|<\infty$, then the series $\sum a_{n}$ is said to be absolutely Nörlund summable or $\left|N, p_{n}\right|$ summable.

Let $f$ be an integrable function, periodic with period $2 \pi$, and its Received 27 April 1970. 
Fourier series be

$$
f(x) \sim \frac{1}{2} a_{0}+\sum_{n=1}^{\infty}\left(a_{n} \cos n x+b_{n} \sin n x\right)=\sum_{n=0}^{\infty} A_{n}(x) .
$$

If the series (2) is $\left|N, p_{n}\right|$ summable, then we say that the Fourier series of $f$ is $\left|N, p_{n}\right|$ summable at the point $x$ and we write $f \in\left|N, p_{n}\right|$.

We use the notations

$$
\begin{gathered}
\varphi(t)=\varphi_{x}(t)=f(x+t)+f(x-t)-2 f(x), \\
\varphi_{1}(t)=\frac{1}{t} \int_{0}^{t} \varphi(u) d u(t>0) .
\end{gathered}
$$

1.2. T. Singh [1] has proved the following theorem (cf. T. Pati [2], H.P. Dikshit [3] and O.P. Varshney [4]):

THEOREM A. If the function $\varphi$ is of bounded variation on the interval $(0, \pi)$ and if the sequence $\left(p_{n}\right)$ is non-increasing and convex, and satisfies the condition

$$
\sum_{k=1}^{n} \frac{P_{k}}{k} \leqq A P_{n} \text { for azz } n \geqq 1,
$$

then $f \in\left|N, p_{n}\right|$.

On the other hand, O.P. Varshney [5] has proved the following:

THEOREM B. If the function $\varphi(t) \log (K / t)(K>\pi)$ is of bounded variation over the interval $(0, \pi)$, then $f \in\left|N, p_{n}\right|$ where

$$
p_{n}=\frac{1}{n+1}\left(1+\frac{1}{2}+\ldots+\frac{1}{n+1}\right) \text { for all } n \geqq 0 \text {. }
$$

T. Pati [6] (of. R. Mohanty and B.K. Ray [7]) has proved that the sequence (4) in Theorem $B$ cannot be replaced by

$$
p_{n}=\frac{1}{n+1} \text { for all } n \geqq 0 \text {. }
$$

The sequence (4) does not satisfy the condition (3) and then Theorem $B$ is not contained in Theorem $A$. 
We shall first prove the following

THEOREM 1. Let $a \geqq 0$ and $K$ be a constant $>\pi$. If the function $\varphi(t)(\log K / t)^{a}$ is of bounded variation over the interval $(0, \pi)$ and if the sequence $\left(p_{n}\right)$ is non-increasing and satisfies the condition

$$
\sum_{k=n}^{\infty} \frac{1}{k P_{k}} \leqq A \frac{(\log n)^{a}}{P_{n}} \text { for all } n>1 \text {, }
$$

then $f \in\left|N, p_{n}\right|$.

The case $a=0$ in Theorem $l$ is a generalization of Theorem A, since the condition of convexity of $\left(p_{n}\right)$ is dropped and the condition (3) implies (5) (see [1]). In the case $a=1$, we get the following:

COROLLARY. If the function $\varphi(t) \log (K / t)$ is of bounded variation on the interval $(0, \pi)$, then $f \in\left|N, p_{n}\right|$, where

$$
p_{n}=\frac{1}{n+1}(\log (n+1))^{b} \text { for alz } n \geqq 0 \text { and some } b>0 \text {. }
$$

The case $b=1$ in the corollary is Theorem $B$ and this corollary does not hold for $b=0$ by Pati's theorem.

1.3. We shall next consider the case that $\varphi_{1}$ is of bounded variation. L.S. Bosanquet [11] has proved the

THEOREM $C$. If the function $\varphi_{1}$ is of bounded variation over the interval $(0, \pi)$, then the Fourier series of $f$ is $\mid C$, a $\mid$ summable at the point $x$ for any $a>1$.

This was generalized by H.P. Dikshit [12] in the following form.

THEOREM D. If the function $\varphi_{1}$ is of bounded variation over the interval $(0, \pi)$ and if the sequence $\left(p_{n}\right)$ is a non-decreasing and concave sequence satisfying the conditions

(i) the sequence $\left((n+1) p_{n} / P_{n}\right)$ is of bounded variation and

$$
\text { (ii) } \sum_{k=n+1}^{\infty} \frac{1}{P_{k}} \leqq A \frac{n}{P_{n}} \text { for all } n \geqq 1 \text {, }
$$

then $f \in\left|n, p_{n}\right|$. 
We shall prove the following generalization.

THEOREM 2. Suppose that the sequence $\left(p_{n}\right)$ is non-decreasing and concave and satisfies the condition

$$
\sum_{k=1}^{\infty} \frac{1}{P_{k}}<\infty .
$$

Then $f \in\left|N, p_{n}\right|$ for any $f$ satisfying the condition that $\varphi_{1}$ is of bounded variation on the interval $(0, \pi)$, if and only if

$$
\sum_{k=n+1}^{\infty}\left|\frac{P_{k-n}}{P_{k}}-\frac{P_{k-n-1}}{P_{k-1}}\right| \leqq A \text { for alz } n \geqq 1 \text {. }
$$

The condition (7) is satisfied when the sequence $\left(P_{n-s} / P_{n}\right)$ is non-decreasing for each $\varepsilon \geqq 1$ or the sequence $\left(p_{n} / P_{n}\right)$ is non-increasing. Further the non-decreasing and concave sequence $\left(p_{n}\right)$ satisfies the following relations which are used in the proof of Theorem 2:

$$
n p_{n} \leqq A P_{n} \text { for all } n \geqq 1 \text {, }
$$

$$
\sum_{n=j+1}^{\infty}\left(p_{n-j}-p_{n-j-1}\right) / P_{n-1} \leqq A / j \text { for all } j \geqq 1 \text {, }
$$

and

$$
p_{n-j+1}-2 p_{n-j}+p_{n-j-1} \geqq 0 \text { for all } j \geqq 1 \text {. }
$$

These are proved easily, so that we omit the proof.

Theorem 2 is a generalization of Theorems $C$ and $D$.

By Theorem 2, we know that $f \in\left|N,(\log (n+1))^{a}\right|(a>1)$ when $\varphi_{1}$ is of bounded variation, since the sequence $p_{n}=(\log (n+1))^{a}(a>1)$ satisfies the conditions. But we don't know the case $a=1$.

1.4. For the proof of Theorem 1 we use the following lemma due to E. Hille and J.D. Tamarkin [8] (see [9]).

LEMMA. If the sequence $\left(p_{n}\right)$ is positive non-increasing, then 


$$
\left|\sum_{k=0}^{N} p_{k} \sin (n-k) t\right| \leqq A P_{[1 / t]} \text { or } \leqq A p_{0} / t
$$

for any $N$, any $n$ and any $t>0$.

\section{Proof of the theorems}

2.1. Proof of Theorem 1: the case $a=0$. By (2) and by integration by parts, we have

$$
A_{j}(x)=\frac{2}{\pi} \int_{0}^{\pi} \varphi(t) \cos j t d t=-\frac{2}{j \pi} \int_{0}^{\pi} \sin j t d \varphi(t)
$$

and we can suppose $A_{0}(x)=0$ without loss of generality. By the formula (1),

$$
-\Delta t_{n-1}=t_{n}-t_{n-1}=\frac{1}{P_{n}{ }^{P} n-1} \sum_{j=1}^{n}\left(P_{n} p_{n-j}-P_{n-j} p_{n}\right) A_{j}(x),
$$

and then

$$
\frac{\pi}{2} \sum_{n=1}^{\infty} \cdot\left|\Delta t_{n}\right| \leqq \int_{0}^{\pi}|d \varphi(t)|\left(\sum_{n=1}^{\infty}\left|\sum_{j=1}^{n} \frac{P_{n} p_{n-j}-P_{n-j} p_{n}}{P_{n} P_{n-1}} \frac{\text { sinjt }}{j}\right|\right) .
$$

It is enough to prove that the sum on the right side is uniformly bounded in $t$. We write, putting $s=[1 / t]$,

$$
\sum_{n=1}^{\infty}\left|\sum_{j=1}^{n} \frac{P_{n} p_{n-j}-P_{n-j} p_{n}}{P_{n}{ }^{P} n-1} \frac{\operatorname{sinjt}}{j}\right|=\sum_{n=1}^{s}+\sum_{n=s+1}^{\infty}=U+V .
$$

Since $P_{n-j} / P_{n} \uparrow 1$ as $n \uparrow \infty$ for each $j$,

$$
\begin{aligned}
U & \leqq t \sum_{n=1}^{s} \sum_{j=1}^{n} \frac{P_{n} p_{n-j}-P_{n-j} P_{n}}{P_{n} P_{n-1}}=t \sum_{j=1}^{s} \sum_{n=j}^{s}\left(\frac{P_{n-j}}{P_{n}}-\frac{P_{n-j-1}}{P_{n-1}}\right) \\
& =t \sum_{j=1}^{s}\left(\frac{s-j}{P_{s}}-\frac{P_{-1}}{P_{j-1}}\right) \leqq A .
\end{aligned}
$$

Now 


$$
\begin{aligned}
V & =\sum_{n=s+1}^{\infty}\left|\sum_{j=1}^{n} \frac{P_{n}{ }^{p} n-j-P_{n-j} p_{n}}{P_{n} P_{n-1}} \frac{\text { sinjt }}{j}\right| \leqq \sum_{n=s+1}^{\infty}\left|\sum_{j=1}^{s}\right|+\sum_{n=s+1}^{\infty}\left|\sum_{j=s+1}^{n}\right|, \\
& =W+X
\end{aligned}
$$

where

$$
\begin{aligned}
W & \leqq \sum_{n=s+1}^{\infty} \sum_{j=1}^{s}\left(\frac{P_{n-j}}{P_{n}}-\frac{P_{n-j-1}}{P_{n-1}}\right)=t \sum_{j=1}^{s} \sum_{n=s+1}^{\infty}\left(\frac{P_{n-j}}{P_{n}}-\frac{P_{n-j-1}}{P_{n-1}}\right) \\
& =t \sum_{j=1}^{s}\left(1-\frac{P_{s-j}}{P_{s}}\right) \leqq A
\end{aligned}
$$

and

$$
\begin{aligned}
X & =\sum_{n=s+1}^{2 s+1}\left|\sum_{j=s+1}^{n}\right|+\sum_{n=2 s+2}^{\infty}\left|\sum_{j=s+1}^{[n / 2]}\right|+\sum_{n=2 s+2}^{\infty}\left|\sum_{j=[n / 2]+1}^{\infty}\right| \\
& =X^{\prime}+Y+Z .
\end{aligned}
$$

$X^{\prime}$ is bounded by the estimation similar to $W$. Writing. $[n / 2]=m$,

$$
\begin{aligned}
& Y=\sum_{n=2 s+2}^{\infty}\left|\sum_{j=s+1}^{m} \frac{P_{n} p_{n-j}-P_{n-j} p_{n}}{j P_{n} P_{n-1}} \frac{\cos (j-1 / 2) t-\cos (j+1 / 2) t}{2 \sin t / 2}\right| \\
& \leqq \sum_{n=2 s+2}^{\infty}\left\{\left|\frac{{ }_{n}^{P} n-m^{-P} n-m^{p} n}{m{ }_{n}^{P} n-1} \frac{\cos (m+1 / 2) t}{2 \sin t / 2}\right|\right. \\
& +\left|\sum_{j=s+1}^{m-1} \Delta\left(\frac{n^{p} n-j^{-P} n-j^{p} n}{j{ }_{n}^{P} n-1}\right) \frac{\cos (j+1 / 2) t}{2 \sin t / 2}\right| \\
& +\left|\frac{P_{n}^{p} n-s-1-P_{n-s-1} p_{n}}{(s+1) P_{n} P_{n-1}} \frac{\cos (s+1 / 2) t}{2 \sin t / 2}\right| \mid \\
& =Y_{1}+Y_{2}+Y_{3},
\end{aligned}
$$

where

$$
\begin{aligned}
& y_{1} \leqq \frac{A}{t} \sum_{n=2 s+2}^{\infty} \frac{1}{n}\left(\frac{P_{n-m}}{P_{n}}-\frac{P_{n-m-1}}{P_{n-1}}\right) \leqq \frac{A}{t} \sum_{n=2 s+2}^{\infty} \frac{P_{m-1}}{n P_{n}}+\frac{A}{t} \sum_{n=2 s+2}^{\infty} \frac{1}{n^{2}} \leqq A, \\
& Y_{2} \leqq \frac{A}{t} \sum_{n=2 s+2}^{\infty} \sum_{j=s+1}^{n-1}\left(\frac{1}{j}\left(\frac{P_{n-j-1}}{P_{n-1}}-\frac{P_{n-1}}{P_{n}}\right)+\frac{1}{j^{2}}\left(\frac{P_{n-j-1}}{P_{n}}-\frac{P_{n-j-2}}{P_{n-1}}\right)\right) \\
& \leqq \frac{A}{t} \sum_{j=s+1}^{\infty} \sum_{n=2 j+1}^{\infty} \leqq \frac{A}{t} \sum_{j=s+1}^{\infty}\left(\frac{P_{j}}{j P_{2 j}}+\frac{1}{j^{2}}\right) \leqq A,
\end{aligned}
$$


and similarly $Y_{3}$ is also bounded. Finally, using the lenma and condition (5) with $a=0$,

$$
\begin{aligned}
z & =\sum_{n=2 s+2}^{\infty} \mid \sum_{j=m+1}^{n}\left(\frac{1}{j P_{n-1}} p_{n-j} \text { sinjt }-\frac{P_{n}}{P_{n} P_{n-1}} \frac{P_{n-j}}{j} \text { sinjt }\right) \mid \\
& \leqq A P_{s} \sum_{n=2 s+2}^{\infty} \frac{1}{n P_{n-1}}+\frac{A}{t} \sum_{n=2 s+2}^{\infty} \frac{P_{n}{ }^{P} n-m-1}{n P_{n} P_{n-1}} \leqq A .
\end{aligned}
$$

Thus we have proved Theorem 1 in the case $a=0$.

2.2. Proof of Theorem 1: the case $a>0$. We put

$$
h(t)=\varphi(t)(\log K / t)^{a} \text { for } 0<t \leqq \pi .
$$

Then, by (2) and by integration by parts,

$$
\begin{aligned}
A_{j}(x) & =\frac{\dot{2}}{\pi} \int_{0}^{\pi} \varphi(t) \cos j t d t \\
& =\frac{2}{\pi} h(\pi) \int_{0}^{\pi} \frac{\cos j u}{(\log K / u)^{a}} d u-\frac{2}{\pi} \int_{0}^{\pi} d h(t) \int_{0}^{t} \frac{\cos j u}{(\log K / u)^{a}} d u .
\end{aligned}
$$

Putting $s=[1 / t]$, we have

$$
\begin{aligned}
\frac{\pi}{2} \sum_{n=1}^{\infty}\left|\Delta t_{n}\right| & =\sum_{n=1}^{\infty}\left|\sum_{j=1}^{n} \frac{P_{n} p_{n-j^{-P}}{ }_{n-j} p_{n}}{P_{n}^{P} n-1} A_{j}(x)\right| \\
& \leqq A|h(\pi)| \sum_{n=1}^{\infty}\left|\sum_{j=1}^{n} \frac{P_{n} p_{n-j}-P_{n-j} p_{n}}{P_{n}{ }^{P} n-1} \int_{0}^{\pi} \frac{\cos j u}{(\log K / u)^{a}} d u\right| \\
& +A \int_{0}^{\pi}|d h(t)|\left(\sum_{n=1}^{s}+\sum_{n=s+1}^{\infty}\right)\left|\sum_{j=1}^{n} \frac{P_{n} p_{n-j}-P_{n-j} p_{n}}{P_{n} P_{n-1}}\right|_{0}^{t} \frac{\cos j u}{(\log K / u)^{a}} d u \mid \\
& =R+s+T .
\end{aligned}
$$

Since the sequence $\left(p_{n}\right)$ is non-increasing and

$$
\left|\int_{0}^{t} \frac{\cos j u}{(\log K / u)^{a}} d u\right| \leqq \frac{t}{(\log K / t)^{a}},
$$

we get 


$$
\begin{aligned}
s & \left.\leqq \int_{0}^{\pi}|d h(t)| \frac{t}{(\log K / t)^{a}} \sum_{n=1}^{s} \sum_{j=1}^{n} \int_{P_{n-j}}^{P_{n}}-\frac{P_{n-j-1}}{P_{n-1}}\right) \\
& \left.=\int_{0}^{\pi} \frac{t|d h(t)|}{(\log K / t)^{a}} \sum_{j=1}^{s} \int^{P} \frac{s-j}{P_{s}}-\frac{-1}{P_{j-1}}\right) \leqq \int_{0}^{\pi} \frac{|d h(t)|}{(\log K / t)^{a}} \leqq A .
\end{aligned}
$$

Now, by integration by parts,

$$
\int_{0}^{t} \frac{\cos j u}{(\log K / u)^{a}} d u=\frac{\sin j t}{j(\log K / t)^{a}}+\frac{a}{j} \int_{0}^{t} \frac{\sin j u}{u(\log K / u)^{a+1}} d u,
$$

and then

$$
\begin{aligned}
T \leqq A \int_{0}^{\pi} \frac{|d n(t)|}{(\log K / t)^{a}}\left(\sum_{n=s+1}^{\infty}\left|\sum_{j=1}^{n} \frac{P_{n} p_{n-j} P_{n-j} p_{n}}{P_{n} P_{n-1}} \frac{\operatorname{sinj} t}{j}\right|\right) \\
\quad+A \int_{0}^{\pi} \cdot|d n(t)|\left(\sum_{n=s+1}^{\infty}\left|\int_{0}^{t}\left(\sum_{j=1}^{n} \frac{P_{n}{ }_{n-j}-P_{n-j} p_{n}}{P_{n} P_{n-1}} \frac{\sin j u}{j}\right) \frac{d u}{u(\log K / u)^{a+1}}\right|\right) \\
=U+V .
\end{aligned}
$$

$U$ is bounded by the estimation of $V$ in the last section, using the condition (5) with $a>0$ and

$$
\begin{aligned}
V & \leqq A \int_{0}^{\pi}|d h(t)|\left(\sum_{n=s+1}^{\infty} \sum_{j=1}^{n}\left(\frac{P_{n-j}}{P_{n}}-\frac{P_{n-j-1}}{P_{n-1}}\right) \frac{1}{j(\log K j)^{a+1}}\right) \\
& \leqq A \int_{0}^{\pi}|d h(t)|\left(\sum_{n=s+1}^{\infty} \sum_{j=1}^{s}+\sum_{n=s+1}^{\infty} \sum_{j=s+1}^{n}\right) \\
& \leqq A \int_{0}^{\pi}|d h(t)|\left(\sum_{j=1}^{s} \frac{1}{j(\log K j)^{a+1}}\left(1-\frac{P_{s-j}}{P_{s}}\right)+\sum_{j=s+1}^{\infty} \frac{j(\log j)^{a+1}}{j(1-}\right) \leqq A .
\end{aligned}
$$

Therefore $T$ is also bounded. It remains to prove $R$ is bounded. Using integration by parts,

$$
\begin{aligned}
R & =A|h(\pi)| \sum_{n=1}^{\infty}\left|\sum_{j=1}^{n} \frac{P_{n} p_{n-j}-p_{n-j} p_{n}}{P_{n}^{P}{ }^{P}} \int_{0}^{\pi} \frac{\sin j t}{j(\log K / t)^{a+1}} d t\right| \\
& \leqq A \sum_{n=1}^{\infty} \sum_{j=2}^{n} \frac{1}{j(\log j)^{a+1}}\left(\frac{P_{n-j}}{P_{n}}-\frac{P_{n-j-1}}{P_{n-1}}\right) \\
& =A \sum_{j=2}^{\infty} \frac{1}{j(\log j)^{a+1}} \sum_{n=j}^{\infty}\left(\frac{{ }^{P} n-j}{P_{n}}-\frac{P_{n-j-1}}{P_{n-1}}\right)=A \sum_{j=2}^{\infty} \frac{1}{j\left(\log j_{j}\right)^{a+1}}<A,
\end{aligned}
$$


by the following inequality

$\left|\int_{0}^{\pi} \frac{\sin j t}{t(\log K / t)^{a+1}} d t\right| \leqq\left|\int_{0}^{\pi / j} \frac{\sin j t}{t(\log K / t)^{a+1}} d t\right| \leqq \frac{A}{(\log j)^{a+1}}$ for all $j \geqq 2$.

This inequality is easily seen from the fact that the function

$t(\log K / t)^{a+1}$ is non-decreasing on the interval $(0, \pi)$, by taking

$K=\pi e^{a+1}$, which does not lose any generality.

Thus Theorem $I$ is completely proved.

2.3. Proof of Theorem 2. We suppose that $\varphi_{1}(\pi)=0$ without any loss of generality. By (2)

$$
A_{j}(x)=\frac{2 j}{\pi} \int_{0}^{\pi} t_{\sin j \varphi_{1}}(t) d t=-\frac{2 j}{\pi} \int_{0}^{\pi} d \varphi_{1}(t) \int_{0}^{t} u \operatorname{sinj} u d u
$$

and then

$$
\begin{aligned}
\frac{\pi}{2} \sum_{n=1}^{\infty}\left|\Delta t_{n}\right| & \leqq \sum_{n=1}^{\infty} \mid \sum_{j=1}^{n} \frac{P_{n} p_{n-j}-P_{n-j} p_{n}}{P_{n}{ }^{P} n-1} \int_{0}^{\pi} d \varphi_{1}(t) \int_{0}^{t} j u \text { sinjudu|} \mid \\
& \left.\leqq \int_{0}^{\pi}\left|d \varphi_{1}(t)\right| \sum_{n=1}^{\infty}\left|\sum_{j=1}^{n} \frac{P_{n}{ }_{n-j}-P_{n-j} p_{n}}{P_{n}{ }^{P} n-1} \int_{0}^{t} j u \sin j u d u\right|\right) . \\
& =\int_{0}^{\pi} R(t)\left|d \varphi_{1}(t)\right| .
\end{aligned}
$$

We shall show that, if $\left(p_{n}\right)$ is non-decreasing and concave and satisfies the condition (6), then $R$ is bounded if and only if (7) holds. By (6), we can suppose that $p_{1}=p_{0}$ and the sequence $\left(p_{n}, n \geqq 1\right)$ is concave. We write

$$
\begin{aligned}
R & =\sum_{n=1}^{\infty}\left|\sum_{j=1}^{n} \frac{P_{n} p_{n-j}-P_{n-j} p_{n}}{P_{n} p_{n-1}} \int_{0}^{t} j u s i n j u d u\right|=\sum_{n=1}^{s}+\sum_{n=s+1}^{\infty} \\
& =S+T,
\end{aligned}
$$

where $s=[1 / t]$. We shall put

$$
x_{j}=\sum_{k=1}^{j} k \int_{0}^{t} u \text { sinkudu, }
$$


then

$$
\left|x_{j}\right| \leqq \sum_{k=1}^{j} k^{2} \int_{0}^{t} u^{2} d u \leqq A j^{3} t^{3}
$$

and $x_{j}$ is bounded uniformly in $t$, since

$$
x_{j}=\frac{t \sin (j+1 / 2) t}{2 \sin t / 2}+\int_{0}^{t} \frac{\sin (j+1 / 2) u}{2 \sin u / 2} d u
$$

Now using Abel's transformation,

$$
\begin{aligned}
S & =\sum_{n=1}^{s} \mid \sum_{j=1}^{n}\left(\frac{p_{n-j}}{P_{n-1}}-\frac{P_{n-j} p_{n}}{P_{n} P_{n-1}}\right)\left(x_{j}-x_{j-1}\right) \\
& \leqq A \sum_{n=1}^{s} \frac{1}{P_{n}}+A t^{3} \sum_{n=1}^{s}\left|\sum_{j=1}^{n-1} j^{3}\left(\frac{p_{n-j}-p_{n-j-1}}{P_{n-1}}+p_{n-j}\left(\frac{1}{P_{n-1}}-\frac{1}{P_{n}}\right)\right)\right| \\
& \leqq A+A t^{3} \sum_{j=1}^{s-1} j^{3}\left(\sum_{n=j+1}^{s} \frac{p_{n-j}-p_{n-j-1}}{P_{n-1}}+\frac{p_{1}}{P_{j}}\right) \leqq A
\end{aligned}
$$

by $(11),(6)$ and (9). On the other hand

$$
\begin{aligned}
T & =\sum_{n=s+1}^{\infty}\left|\frac{P_{0} x_{n}}{P_{n}}+\sum_{j=1}^{n-1}\left(\frac{p_{n-j}-p_{n-j-1}}{P_{n-1}}-\frac{p_{n-j} p_{n}}{P_{n} P_{n-1}}\right) x_{j}\right| \\
& \leqq A \sum_{n=s+1}^{\infty} \frac{1}{P_{n}}+\sum_{n=s+1}^{\infty}\left|\sum_{j=1}^{s}\right|+\sum_{n=s+1}^{\infty}\left|\sum_{j=s+1}^{n-1}\right|=A+U+V,
\end{aligned}
$$

where

$$
U \leqq A t^{3} \sum_{j=1}^{s} j^{3} \sum_{n=s+1}^{\infty}\left(\frac{p_{n-j}-p_{n-j-1}}{P_{n-1}}+p_{n-j}\left(\frac{1}{P_{n-1}}-\frac{1}{P_{n}}\right)\right) \leqq A
$$

by (11) and (9), and by (12) we can write

$$
\begin{aligned}
V \leqq A & \sum_{n=s+1}^{\infty}\left|\sum_{j=s+1}^{n-1}\left(\frac{p_{n-j}-p_{n-j-1}}{P_{n-1}}-\frac{p_{n-j} p_{n}}{P_{n} P_{n-1}}\right) \sin (j+1 / 2) t\right| \\
& \left.+\sum_{n=s+1}^{\infty} \mid \int_{0}^{t} \frac{d u}{2 s i n u / 2} \sum_{j=s+1}^{n-1}\left(\frac{p_{n-j}-p_{n-j-1}}{P_{n-1}}-\frac{p_{n-j} p_{n}}{P_{n} P_{n-1}}\right) \sin (j+1 / 2) u\right) \mid \\
& =A W+X .
\end{aligned}
$$

Now again using Abel's transformation, we get 


$$
\begin{aligned}
(2 \sin t / 2) W= & \sum_{n=s+1}^{\infty}\left|\sum_{j=s+1}^{n-1}\left(\frac{P_{n-j}-p_{n-j-1}}{P_{n-1}}-\frac{p_{n-j} p_{n}}{P_{n} P_{n-1}}\right)(\cos j t-\cos (j+1) t)\right| \\
= & \sum_{n=s+1}^{\infty}\left(\frac{P_{0}}{P_{n-1}}-\frac{p_{1}}{P_{n}}\right) \\
& \quad+\sum_{n=s+1}^{\infty} \sum_{j=s+2}^{n-1}\left(\frac{p_{n-j-1}-2 p_{n-j}+p_{n-j+1}}{P_{n-1}}+\frac{\left(p_{n-j+1}-p_{n-j}\right) p_{n}}{P_{n} P_{n-1}}\right) \\
& +\sum_{n=s+1}^{\infty}\left(\frac{p_{n-s-1}-p_{n-s-2}}{P_{n-1}}+\frac{p_{n-s-1} p_{n}}{P_{n} P_{n-1}}\right) \\
= & Y_{1}+y_{2}+Y_{3}
\end{aligned}
$$

by $(10)$, where

$$
Y_{1} \leqq A \sum_{n=s+1}^{\infty}\left(\frac{1}{P_{n-1}}-\frac{1}{P_{n}}\right) \leqq \frac{A}{P_{s}} \leqq A t
$$

by (8),

$$
\begin{aligned}
y_{2} & \leqq A \sum_{n=s+1}^{\infty} \sum_{j=s+2}^{n-1} \frac{p_{n-j-1}-2 p_{n-j}+p_{n-j+1}}{P_{n-1}} \leqq A \sum_{n=s+1}^{\infty} \frac{p_{n-s-1}-p_{n-s-2}}{P_{n-1}} \\
& \leqq A t
\end{aligned}
$$

by (9) and similarly $Y_{3} \leqq A t$. Therefore $W$ is bounded, so that it remains to estimate $X$. We can easily see that

$$
\int_{0}^{t} \frac{\sin (j+1 / 2) u}{2 \sin u / 2} d u=\frac{\pi}{2}-\int_{t}^{\pi} \frac{\sin (j+1 / 2) u}{2 \sin u / 2} d u=\frac{\pi}{2}-y_{j}
$$

where $\left|y_{j}\right| \leqq A / j t$, and hence

$$
\begin{aligned}
X= & \left.\sum_{n=s+1}^{\infty}\left|\int_{0}^{t} \frac{d u}{2 \sin u / 2}\right| \sum_{j=s+1}^{n-1}\left(\frac{P_{n-j}-p_{n-j-1}}{P_{n-1}}-\frac{p_{n-j} p_{n}}{P_{n-1} P_{n}}\right) \sin (j+1 / 2) u\right) \mid \\
\leqq & \sum_{n=s+1}^{\infty}\left|\sum_{j=s+1}^{n-1}\left(\frac{p_{n-j} p_{n-j-1}}{P_{n-1}}-\frac{p_{n-j} p_{n}}{P_{n-1} P_{n}}\right)\right| \\
& +\frac{A}{t} \sum_{n=s+1}^{\infty}\left(\sum_{j=s+1}^{n-1} \frac{p_{n-j}-p_{n-j-1}}{j P_{n-1}}+\sum_{j=s+1}^{n-1} \frac{p_{n-j} p_{n}}{P_{n-1} P_{n}}\right)=\frac{\pi}{2} X_{1}+X_{2}
\end{aligned}
$$

where 


$$
\begin{aligned}
X_{2} & \leqq \frac{A}{t} \sum_{j=s+1}^{\infty} \frac{1}{j}\left(\sum_{n=j+1}^{\infty} \frac{p_{n-j}-p_{n-j-1}}{P_{n-1}}+\sum_{n=j+1}^{\infty} p_{n-j}\left(\frac{1}{P_{n-1}}-\frac{1}{P_{n}}\right)\right) \\
& \leqq \frac{A}{t} \sum_{j=s+1}^{\infty} \frac{1}{j^{2}}+\frac{A}{t} \sum_{j=s+1}^{\infty} \frac{1}{j P_{j}} \leqq A
\end{aligned}
$$

by Abel's transformation, (6) and (9) and

$$
\begin{aligned}
X_{1} & =\sum_{n=s+1}^{\infty}\left|\frac{P_{n-s-1}-p_{0}}{P_{n-1}}-\left(P_{n-s-1}-p_{0}\right) \frac{p_{n}}{P_{n-1} P_{n}}\right| \\
& =\sum_{n=s+1}^{\infty}\left|\frac{n-s-1}{P_{n}}-\frac{P_{n-s-2}}{P_{n-1}}\right|+0\left(\sum_{n=s+1}^{\infty} \frac{1}{P_{n}}\right) .
\end{aligned}
$$

Thus we have proved that

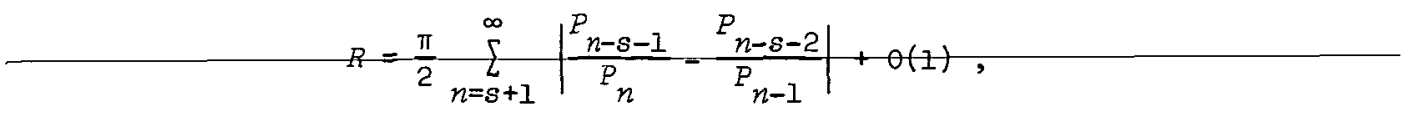

by (6), therefore the condition (7) is necessary and sufficient for boundedness of $R$. Thus Theorem 2 is proved.

\section{References}

[1] Tarkeshwar Singh, "Absolute Nörlund summability of Fourier series", Indian J. Math. 6 (1964), 129-136.

[2] T. Pati, "On the absolute Nörlund summability of a Fourier series", J. London Math. Soc. 34 (1959), 153-160. "Addendum: On the absolute Nörlund summability of a Fourier series", J. London Math. Soc. 37 (1962), 256.

[3] H.P. Dikshit, "Absolute summability of a Fourier series by Nörlund means", Math. Z. 102 (1.967), 166-170.

[4] O.P. Varshney, "On the absolute Nörlund summability of a Fourier series", Math. Z. 83 (1964), 18-24.

[5] O.P. Varshney, "On the absolute summability of Fourier series by a Nörlund method", Univ. Roorkee Res. J. 6 (1963), 103-113.

[6] T. Pati, "The non-absolute summability of Fourier series by a Nörlund method", Indian M. Math. 25 (1961), 197-214. 
[7] R. Mohanty and B.K. Ray, "On the non-absolute summability of a Fourier series and the conjugate of a Fourier series by a Nörlund method", Proc. Cambridge Philos. Soc. 63 (1967), $407-411$.

[8] Einar Hille and J.D. Tamarkin, "On the summability of Fourier series I", Trans. Amer. Math. Soc. 34 (1932), 757-783.

[9] Leonard McFadden, "Absolute Nörlund summability", Duke Math. J. 9 (1942), 168-207.

[10] A. Zygmund, Trigonometric series (Cambridge University Press, 2nd ed., Vol. 1, New York, 1959).

[11] L.S. Bosanquet, "The absolute Cesàro summability of a Fourier series", Proc. London Math. Soc. (2) 41 (1936), 517-528.

[12] H.P. Dikshit, "Absolute $(C, I) \cdot\left(N, p_{n}\right)$ summability of a Fourier series and its conjugate series", Pacific J. Math. 26 (1968), $245-256$.

Institute of Advanced Studies, Australian National University, Canberra, ACT. 